Research Paper

\title{
Prognostic Influence of the Time Interval between Surgery and Chemotherapy in Epithelial Ovarian Cancer
}

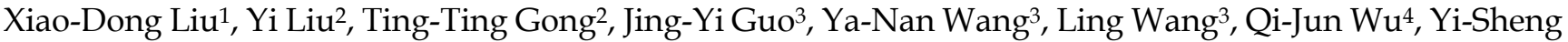 \\ $\mathrm{JiaO}^{2 \bowtie}$ \\ 1. Department of Pharmacy, Shengjing Hospital of China Medical University, Shenyang, China \\ 2. Department of Obstetrics and Gynecology, Shengjing Hospital of China Medical University, Shenyang, China \\ 3. School of postgraduate, China Medical University, Shenyang, China \\ 4. Department of Clinical Epidemiology, Shengjing Hospital of China Medical University, Shenyang, China \\ $\triangle$ Corresponding author: Yi-Sheng Jiao, M.D., Ph.D. Department of Obstetrics and Gynecology, Shengjing Hospital of China Medical University, No.36, \\ SanHao Street, Shenyang, Liaoning 110004, P. R. China. Phone: +86-24-96615-41211; Fax: +86-24-96615-41211; E-mail: jiaoys@sj-hospital.org \\ (C) Ivyspring International Publisher. This is an open access article distributed under the terms of the Creative Commons Attribution (CC BY-NC) license \\ (https://creativecommons.org/licenses/by-nc/4.0/). See http://ivyspring.com/terms for full terms and conditions.
}

Received: 2018.05.22; Accepted: 2018.08.20; Published: 2018.10.18

\begin{abstract}
The influence of time to chemotherapy (TTC) on recurrence and survival among epithelial ovarian cancer (EOC) patients still remains unknown. This single center retrospective cohort study was conducted on $489 \mathrm{EOC}$ patients who underwent surgery followed by taxane- plus platinum-based chemotherapy in the Shengjing Hospital of China Medical University between 2011 and 2015. The Multivariate cox proportional regression models were used to calculate hazard ratios (HRs) and 95\% confidence intervals (Cls) for progression-free survival (PFS) and overall survival (OS) after adjustment for potential confounders. The median follow-up duration was 2.97 years (inter-quartile range from 2.11 to 4.13 years). The recurrence and mortality rate of the all patients was $50.9 \%$ (249/489) and 43.6\% (213/489), respectively. Having comorbidity, residual disease, ascites, and advanced FIGO stage (III-IV) was associated worse PFS and OS of EOC patients. Compared to TTC less than 14 days, delayed TTC (more than 28 days) was associated with a worse PFS (HR=1.36; 95\%Cl: 0.96-1.92) and OS (HR=1.38; 95\% Cl: 0.95-2.00). Notably, in EOC patients with advanced stage, delayed TTC (more than 28 days) was associated with worse PFS (HR=1.51; 95\%Cl: 1.02-2.24) and OS ( $\mathrm{HR}=1.53 ; 95 \% \mathrm{Cl}: 1.01-2.32)$ when comparing to TTC less than 14 days. In conclusion, delayed TTC was associated with higher rates of EOC recurrence and survival among these patients with advanced stage. The findings of the present study may provide evidence for gynecologist as well as these ovarian cancer patients to make further decision for the treatment.
\end{abstract}

Key words: epithelial ovarian cancer; retrospective cohort; survival; time to chemotherapy

\section{Introduction}

Epithelial ovarian cancer (EOC) is the most lethal gynecologic malignancy, with nearly 238,700 cases diagnosed annually worldwide resulting in 151,900 deaths in 2012 [1]. The American Cancer Society has estimated that there will be 22,280 new cases of ovarian cancer and 14,240 deaths during 2016 in the United States [2]. Residual disease (RD) after initial surgery, International Federation of Gynecology and Obstetrics (FIGO) stage, tumor grade, and histologic types are the well-established prognostic factors influencing the response rate to chemotherapy and survival rate of this disease [3]. Platinum-based chemotherapy is routinely recommended after primary surgery aiming at complete tumor resection for advanced ovarian cancer. In routine clinical practice, a lot of discussion is focused on the optimal time from surgery to the start of chemotherapy in this disease, however, this question has not been well established so far.

Previous experimental studies suggested that removal of the primary tumor may increase the numbers of circulating tumor cells and potentiate the 
growth of metastatic deposits [4-9]. This increase in metastatic growth is probably thought to correlate with a reduction in angiogenesis inhibitors, such as angiostatin, following surgery $[4,5,9]$. Furthermore, in vivo studies suggest a decreased survival after a longer time to chemotherapy (TTC) as increased metastatic growth after surgery was found $[5,6,10]$. Despite these emerging biological evidence, the optimal time between primary surgery and initiation of chemotherapy has been controversial in the results of published epidemiological studies [11-23]. Warwick et al [23] first reported the TTC was positively associated with overall survival (OS) on the basis of two prospective randomized phase III trials in 1995. Subsequently, several prospective studies as well as retrospective studies found similar positive results of aforementioned association. For example, Hofstetter et al [16] analyzed the data of 191 patients with advanced serous ovarian cancer from a prospective multicenter study OVarian CAncer Diagnosis and suggested that compared to patients who received the first cycle of chemotherapy $>28$ days after surgery, patients with an earlier ( $\leq 28$ days) start of chemotherapy had a significantly improved 3-year survival rate of $73 \%$ after adjustment for several potential confounders. However, negative findings were also observed in some studies $[11,13,18,19,21$, 22]. A most recent report from China found non-significant results whether patients were categorized into four groups by TTC quartile or two groups. Further stratified by with and without RD, there were still no differences in progression-free survival (PFS) and OS [11].

These inconsistent results might be attributed to different inclusion criteria of patients, TTC category, and whether adjustment for potential confounders of these studies [24]. Herein, to evaluate whether the length of the interval from primary surgery to platinum-based chemotherapy in relation to the survival of patients with EOC, we reported the aforementioned association in a retrospective study which was carried out in the Shengjing Hospital of China Medical University. The findings of the present study may provide evidence for gynecologist as well as these ovarian cancer patients to make further decision for the treatment.

\section{Patients and Methods}

\section{Study patients}

This retrospective study was conducted at the Shengjing Hospital of China Medical University, Shenyang, China between December 1, 2011 and December 31, 2015. Patients were included if they were diagnosed as primary EOC and received taxane- plus platinum (cisplatin or carboplatin)-based intravenous chemotherapy. In contrast, patients were excluded if they underwent surgical exploration at other institution but received chemotherapy in the Shengjing hospital, received neoadjuvant therapy or non taxane- plus platinum-based chemotherapy, receive intraperitoneal chemotherapy, and were treated for recurrent disease. The study was approved by the Institutional Review Board of Shengjing Hospital of China Medical University (2015PS38K).

\section{Data collection}

TTC was defined as the time interval between the primary surgery and initiation of chemotherapy. Information on demographic and clinical factors was obtained through patients' electronic medical records from hospital information system of the Shengjing hospital. Data included date at diagnosis, date of surgery, date of chemotherapy, tumor histology, tumor grade, comorbidity, RD, ascites, and treatment.

Tumor stage and grade was calculated according to criteria of the FIGO and the histologic typing system of the $\mathrm{WHO}$, respectively. Tumors were graded as well $\left(\mathrm{G}_{1}\right)$, moderately $\left(\mathrm{G}_{2}\right)$, or poorly $\left(\mathrm{G}_{3}\right)$ differentiated. $\mathrm{RD}$ was divided into either "none detectable' when none visible disease was left at the end of surgery. If visible disease was left, we classified them into ' $\leq 1 \mathrm{~cm}$ ' and ' $>1 \mathrm{~cm}$ ' according the size of the disease. Performance status (PS) was evaluated according to the criteria of the Eastern Cooperative Oncology Group's (ECOG) scale. Comorbidity, which is defined as the presence of one or more diseases in addition to the primary disease, was classified as 'yes (score $\geq 1)^{\prime}$ or 'no (score $=0$ )' using the Charlson comorbidity index. All these aforementioned information were collected and checked by two experienced gynecologists and pathologists.

In accordance to Response Evaluation Criteria in Solid Tumors (RECIST) criteria [25], the evaluation of the clinical progression of disease was based on clinical examination, serum CA-125 assay, chest $x$-ray, abdominal-pelvic ultrasound, and computed tomography scan. Additional investigations were performed when appropriate. The primary endpoint was PFS, defined as time from the completion of primary surgery to first progression or recurrence of disease or death from any cause. OS was defined as time from the completion of primary surgery to death from any cause or date of last follow-up (December $31,2017)$ for patients still alive. Cause of death was obtained from the death certificates.

\section{Statistical Analyses}

Descriptive statistics were used for demographic and clinical characteristics across TTC categories and 
evaluated using Mann-Whitney $U$ test for continuous variables and Chi-square test for categorical variables. Continuous variables were summarized as the median with inter-quartile range (IQR). Categorical variables were expressed as number with percent. The Cox Proportional Hazards Model was applied to estimate hazard ratios (HRs) and 95\% confidence intervals (CIs). We assessed the proportional hazards assumption with a likelihood ratio test. TTC was categorized into three groups: less than 14 days, between 14 to 28 days, and more than 28 days.

Tests for linear trend were performed by assigning an ordinal value (1, 2, and 3$)$ to each category of exposure and treating it as a continuous variable in the regression model. Additionally, we applied the restricted cubic spline function (three knots) in Cox regression analyses to assess the possible nonlinear association between TTC and PFS and OS [26-28]. The log-rank test was used to compare the homogeneity of survival functions across strata defined by categories of TTC.

We conducted multivariable adjusted analyses, including the following potential confounders: age at diagnosis, FIGO stage, RD, comorbidity, performance status, ascites, and cancer grading. Furthermore, we carried out subgroup analyses stratified by these aforementioned potential confounders. Likelihood ratio tests were conducted to examine whether the associations between TTC and PFS and OS were modified by the following pre-specified potential effect modifiers: FIGO stage, RD, comorbidity, performance status, ascites, and cancer grading. In sensitivity analyses, we excluded: patients failed to finish six cycles of platinum-based intravenous chemotherapy $(n=39)$ or patients who recurred or died within 1 year of study enrollment $(n=37)$. All analyses were performed by SAS software (version 9.3; SAS Institute, Cary, NC).

\section{Results}

One thousand three hundred and ninety women with ovarian, primary peritoneal cancers, and fallopian tube cancers were identified in the Shengjing hospital from December 1, 2011 to December 31, 2015. We excluded 47 women who were diagnosed as primary peritoneal cancers and fallopian tube cancers, 159 patients received neoadjuvant chemotherapy, 221 patients did not undergo primary surgery/ underwent surgical exploration at other institution, 14 patients aged younger than 15, 460 patients had missing information in any variables or covariates. Finally, of the 489 patients included in the analysis, the median age of these patients was 53 (IQR: 48 to 59). After a median observation period of 2.97 years (IQR: 2.11 to 4.13 years), 249 (50.9\%) and 213 (43.6\%) patients were recurred and died, respectively. The median interval of TTC was 11 days, the IQR was 8 to 25 days.

Table 1 presents the demographic characteristics and clinical predictors of these patients according to TTC. Among these patients with delayed TTC, EOC patients with non-serous histology were more common than patients with TTC less than 14 days $(\mathrm{P}<0.05)$. However, there is no significant difference among other demographic characteristics and clinical predictors. Table 2 summarizes selected patient characteristics in relation to PFS and OS after mutual adjustment for each other. Having comorbidity, RD, ascites, and advanced FIGO stage (III-IV) was associated poor PFS and OS of EOC patients.

Table 1. Demographic characteristics and clinical predictors of epithelial ovarian cancer patients according to time interval between surgery and chemotherapy

\begin{tabular}{|c|c|c|c|c|}
\hline \multirow[t]{2}{*}{ Variables } & \multicolumn{3}{|c|}{$\begin{array}{l}\text { Time interval between surgery } \\
\text { and chemotherapy (days) }\end{array}$} & \multirow[t]{2}{*}{$\begin{array}{l}P \text { value } \\
\dagger\end{array}$} \\
\hline & $\leq 14$ & $14-28$ & $>28$ & \\
\hline No. of patients & 300 & 95 & 94 & \\
\hline $\begin{array}{l}\text { Age at diagnosis (years), } \\
\text { Median (inter-quartile range) }\end{array}$ & $53(48-59)$ & $51(49-59)$ & $54.5(48-61)$ & \\
\hline $\begin{array}{l}\text { Follow-up time (years), } \\
\text { Median (inter-quartile range) }\end{array}$ & $\begin{array}{l}3.0 \\
(2.1-4.4)\end{array}$ & $\begin{array}{l}2.9 \\
(1.9-3.9)\end{array}$ & $2.7(2.3-3.8)$ & \\
\hline Vital status $(\%)$ & & & & 0.10 \\
\hline Alive & $158(52.7)$ & $60(63.2)$ & $58(61.7)$ & \\
\hline Died & $142(47.3)$ & $35(36.8)$ & $36(38.3)$ & \\
\hline Recurrence status (\%) & & & & 0.69 \\
\hline Yes & $149(49.7)$ & $52(54.7)$ & $48(51.1)$ & \\
\hline No & $151(50.3)$ & $43(45.3)$ & $46(48.9)$ & \\
\hline Histology (\%) & & & & $<0.05$ \\
\hline Serous & $238(79.3)$ & $69(72.6)$ & $63(67)$ & \\
\hline Non-serous & $62(20.7)$ & $26(27.4)$ & $31(33)$ & \\
\hline Comorbidity (\%) & & & & 0.67 \\
\hline No & $151(50.3)$ & $52(54.7)$ & $51(54.3)$ & \\
\hline Yes & 149 (49.7) & $43(45.3)$ & $43(45.7)$ & \\
\hline Performance status (\%) & & & & 0.19 \\
\hline 0 & 0 & 0 & $1(1.1)$ & \\
\hline 1 & $73(24.3)$ & $18(19)$ & $26(27.7)$ & \\
\hline 2 & $158(52.7)$ & 49 (51.6) & $39(41.5)$ & \\
\hline$\geq 3$ & $69(23)$ & $28(29.5)$ & $28(29.8)$ & \\
\hline FIGO stage ( $\%)$ & & & & 0.22 \\
\hline I & $55(18.3)$ & $24(25.3)$ & $28(29.8)$ & \\
\hline II & $48(16)$ & $18(19)$ & $11(11.7)$ & \\
\hline III & $181(60.3)$ & $48(50.5)$ & $49(52.1)$ & \\
\hline IV & $16(5.3)$ & $5(5.3)$ & $6(6.4)$ & \\
\hline Residual disease (\%) & & & & 0.08 \\
\hline None detectable & $168(56)$ & $67(70.5)$ & $61(64.9)$ & \\
\hline$\leq 1 \mathrm{~cm}$ & $64(21.3)$ & $12(12.6)$ & $13(13.8)$ & \\
\hline$>1 \mathrm{~cm}$ & $68(22.7)$ & $16(16.9)$ & $20(21.3)$ & \\
\hline Ascites (\%) & & & & 0.13 \\
\hline No & $125(41.7)$ & $45(47.4)$ & $31(33)$ & \\
\hline Yes & $175(58.3)$ & $50(52.6)$ & $63(67)$ & \\
\hline Grading (\%) & & & & 0.50 \\
\hline Grade 1 & $12(4)$ & $4(4.2)$ & $2(2.1)$ & \\
\hline Grade 2 & $81(27)$ & $24(25.3)$ & $33(35.1)$ & \\
\hline Grade 3 & 207 (69) & $67(70.5)$ & $59(62.8)$ & \\
\hline
\end{tabular}

$\dagger$ The Mann-Whitney $U$ and Chi-square test was used for comparing continuous and category variables, respectively. 
Table 2. Demographic and clinical characteristics and progression-free survival and overall survival among epithelial ovarian cancer patients

\begin{tabular}{|c|c|c|c|c|}
\hline \multirow[t]{2}{*}{ Variables } & \multicolumn{2}{|l|}{ PFS } & \multicolumn{2}{|l|}{ OS } \\
\hline & No./Events & HR $(95 \% C I) \dagger$ & No./Events & HR $(95 \% C I) \dagger$ \\
\hline \multicolumn{5}{|l|}{ Age at diagnosis } \\
\hline$\leq 50$ & $191 / 90$ & 1.00 (Ref) & $191 / 78$ & 1.00 (Ref) \\
\hline$>50$ & $298 / 150$ & $1.09(0.83-1.43)$ & $298 / 135$ & $1.19(0.90-1.57)$ \\
\hline \multicolumn{5}{|l|}{ Comorbidity } \\
\hline No & $254 / 107$ & 1.00 (Ref) & $254 / 91$ & 1.00 (Ref) \\
\hline Yes & $235 / 133$ & $1.53(1.18-1.98)$ & $235 / 122$ & $1.70(1.28-2.25)$ \\
\hline \multicolumn{5}{|l|}{ Histology } \\
\hline Serous & $370 / 202$ & 1.00 (Ref) & $370 / 179$ & 1.00 (Ref) \\
\hline Non-serous & $119 / 38$ & $0.77(0.54-1.10)$ & $119 / 34$ & $0.81(0.55-1.19)$ \\
\hline \multicolumn{5}{|l|}{ PS } \\
\hline $0-1$ & $124 / 62$ & 1.00 (Ref) & $124 / 50$ & 1.00 (Ref) \\
\hline 2 & $246 / 107$ & $0.95(0.69-1.31)$ & $246 / 98$ & $1.08(0.75-1.55)$ \\
\hline$\geq 3$ & $119 / 71$ & $1.20(0.82-1.75)$ & $119 / 65$ & $1.24(0.83-1.86)$ \\
\hline \multicolumn{5}{|l|}{ FIGO stage $(\%)$} \\
\hline I-II & $184 / 45$ & 1.00 (Ref) & $184 / 35$ & 1.00 (Ref) \\
\hline III & $278 / 174$ & $2.34(1.62-3.37)$ & $278 / 155$ & $2.60(1.73-3.91)$ \\
\hline IV & $27 / 21$ & $6.52(3.78-11.25)$ & $27 / 23$ & $9.66(5.37-17.38)$ \\
\hline \multicolumn{5}{|l|}{ Residual disease } \\
\hline None detectable & $296 / 107$ & 1.00 (Ref) & $296 / 84$ & 1.00 (Ref) \\
\hline$\leq 1 \mathrm{~cm}$ & $89 / 59$ & $1.67(1.19-2.34)$ & $89 / 57$ & $2.01(1.41-2.87)$ \\
\hline$>1 \mathrm{~cm}$ & $104 / 74$ & $1.96(1.42-2.70)$ & $104 / 72$ & $2.25(1.60-3.17)$ \\
\hline \multicolumn{5}{|l|}{ Ascites } \\
\hline No & $201 / 117$ & 1.00 (Ref) & $201 / 109$ & 1.00 (Ref) \\
\hline Yes & $288 / 123$ & $1.31(0.98-1.75)$ & $288 / 104$ & $1.49(1.11-2.00)$ \\
\hline \multicolumn{5}{|l|}{ Grading } \\
\hline Grade 1 & $18 / 8$ & 1.00 (Ref) & $18 / 6$ & 1.00 (Ref) \\
\hline Grade 2 & $138 / 64$ & $1.08(0.81-1.45)$ & $138 / 54$ & $1.27(0.92-1.75)$ \\
\hline Grade 3 & $333 / 168$ & $1.33(0.65-2.73)$ & $333 / 153$ & $1.71(0.84-3.49)$ \\
\hline
\end{tabular}

CI, confidence interval; FIGO, International Federation of Gynecology and Obstetrics; HR, hazard ratio; OS, overall survival; PFS, progression-free survival.

† HRs (95\% CIs) for progression-free survival and overall survival were estimated by using multivariable proportional hazards models, mutually adjusted for all other variables listed in the table.

Compared with patients with TTC less than 14 days, those with TTC more than 28 days experienced an adjusted hazard ratio (HR) for PFS of 1.36 (95\% CI=0.96-1.92; $P$ for trend=0.08) (Table 3, Figure 1). In our stratified analyses, some of the associations became stronger in selected subgroups, while the directions of the associations were unchanged; there was no significant interaction effect. Of note, we

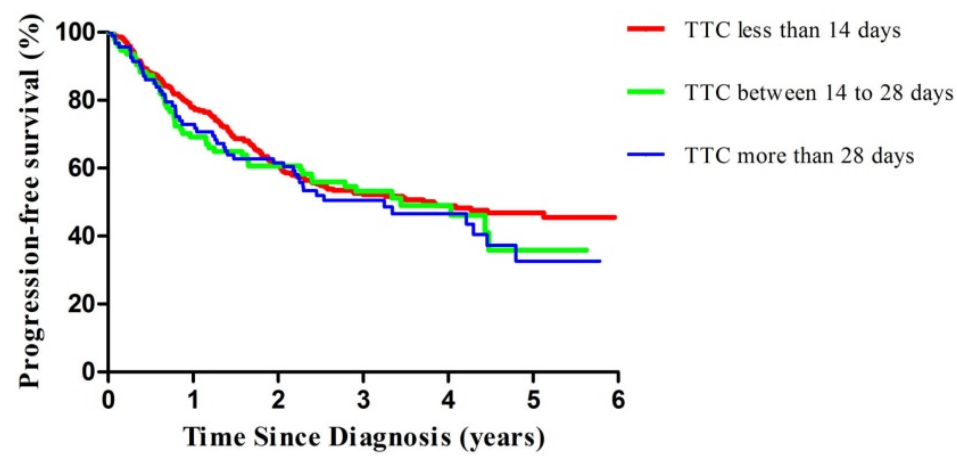

Figure 1. Multivariable-adjusted progression-free survival curves of patients with ovarian cancer by TTC, estimated from a proportional hazards model (adjusted for age at diagnosis, International Federation of Gynecology and Obstetrics, residual disease, performance status, ascites, and grading) by using a direct adjustment method. The red line indicates the TTC less than 14 days, the green line indicates the TTC between 14 to 28 days, and the blue line indicates the TTC more than 28 days. TTC, time to chemotherapy. observed significant results in EOC patients with advanced stage $(\mathrm{HR}=1.51,95 \% \mathrm{CI}=1.02-2.24 ; P$ for trend $<0.05$ ). Similar patterns were observed in the analysis of OS. Compared with patients with TTC less than 14 days, those with TTC more than 28 days experienced an adjusted hazard ratio (HR) for OS of 1.38 (95\%CI=0.95-2.00; $P$ for trend=0.09) (Table 4, Figure 2). Furthermore, we only observed significant results in EOC patients with advanced stage $(\mathrm{HR}=1.53,95 \% \mathrm{CI}=1.01-2.32 ; P$ for trend $<0.05)$.

In sensitivity analyses, we excluded 69 patients who failed to finish the six cycles of chemotherapy, the PFS (HR=1.32, 95\% CI= 1.00-1.74) and $\mathrm{OS}(\mathrm{HR}=1.34,95 \% \mathrm{CI}=1.02-1.76)$ showed robust and statistical significance. Furthermore, we excluded 122 patients who recurred within 1 year of study enrollment or 46 patients who died within 1 year of study enrollment, the PFS and OS were similar to the main findings. Nonlinear dose-response analyses were also conducted among these patients stratified by aforementioned variables, but there was no evidence of a nonlinear association between TTC and PFS and OS of ovarian cancer (data not shown). 
Table 3. HR $(95 \% \mathrm{Cl})$ for progression-free survival among epithelial ovarian cancer patients according to the time interval between surgery and chemotherapy

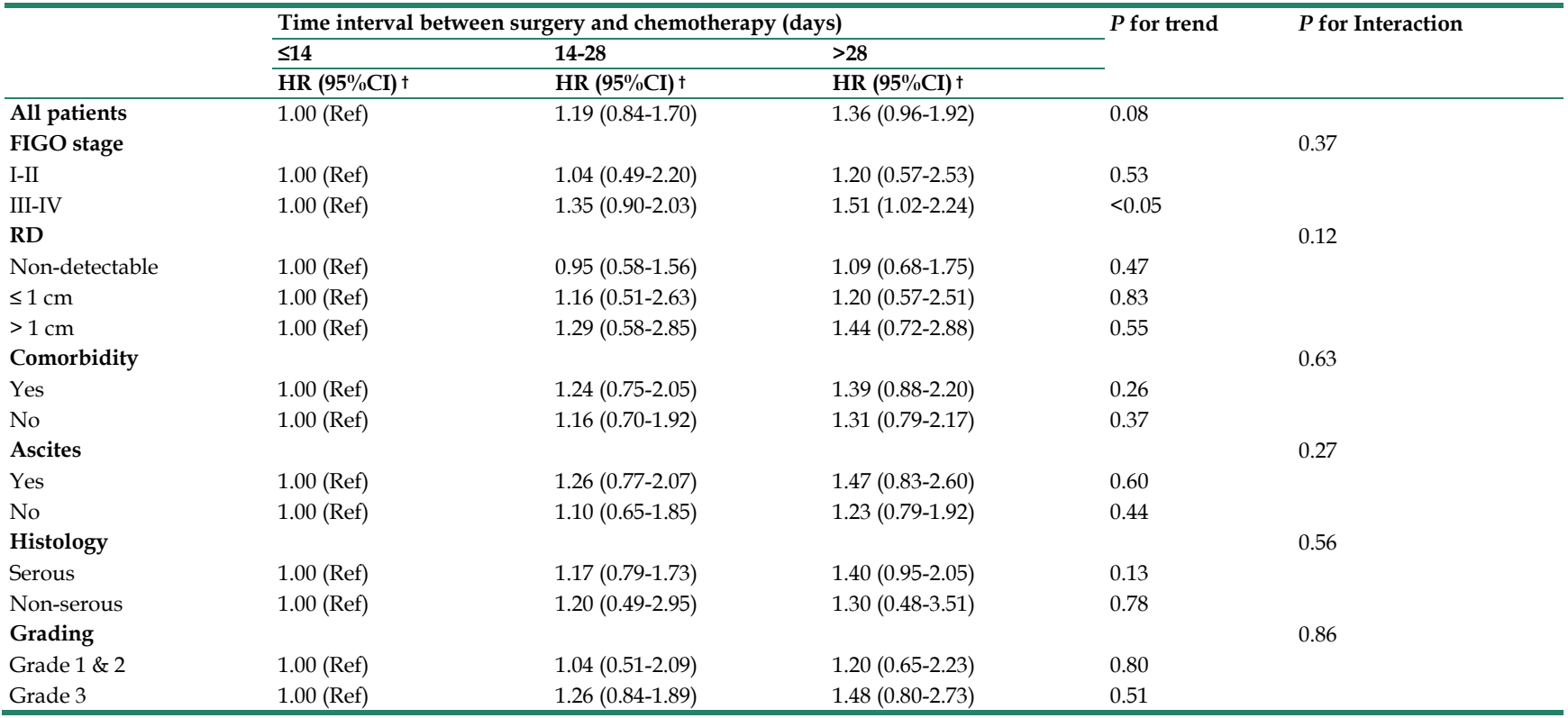

$\mathrm{CI}$, confidence interval; FIGO, International Federation of Gynecology and Obstetrics; HR, hazard ratio; RD, residual disease.

† HRs (95\% CIs) for progression-free survival was estimated by using multivariable proportional hazards models which were adjusted for age at diagnosis, FIGO, RD, performance status, ascites, and grading.

Table 4. HR $(95 \% \mathrm{Cl})$ for overall survival among epithelial ovarian cancer patients according to the time interval between surgery and chemotherapy

\begin{tabular}{|c|c|c|c|c|c|}
\hline & \multicolumn{3}{|c|}{ Time interval between surgery and chemotherapy (days) } & \multirow[t]{3}{*}{$P$ for trend } & \multirow[t]{3}{*}{$P$ for Interaction } \\
\hline & $\leq 14$ & $14-28$ & $>28$ & & \\
\hline & HR $(95 \%$ CI $) \dagger$ & HR $(95 \% \mathrm{CI}) \dagger$ & HR $(95 \% \mathrm{CI}) \dagger$ & & \\
\hline All patients & 1.00 (Ref) & $1.22(0.83-1.79)$ & $1.38(0.95-2.00)$ & 0.09 & \\
\hline FIGO stage & & & & & 0.44 \\
\hline I-II & 1.00 (Ref) & $1.09(0.48-2.48)$ & $1.23(0.36-4.20)$ & 0.69 & \\
\hline III-IV & 1.00 (Ref) & $1.28(0.82-2.00)$ & $1.53(1.01-2.32)$ & $<0.05$ & \\
\hline RD & & & & & 0.19 \\
\hline Non-detectable & 1.00 (Ref) & $0.93(0.52-1.64)$ & $1.07(0.60-1.91)$ & 0.42 & \\
\hline$\leq 1 \mathrm{~cm}$ & 1.00 (Ref) & $1.11(0.49-2.53)$ & $1.20(0.53-2.74)$ & 0.89 & \\
\hline$>1 \mathrm{~cm}$ & 1.00 (Ref) & $1.33(0.58-3.05)$ & $1.52(0.73-3.16)$ & 0.61 & \\
\hline Comorbidity & & & & & 0.58 \\
\hline Yes & 1.00 (Ref) & $1.30(0.78-2.18)$ & $1.45(0.88-2.38)$ & 0.24 & \\
\hline No & 1.00 (Ref) & $1.10(0.63-1.92)$ & $1.29(0.70-2.37)$ & 0.41 & \\
\hline Ascites & & & & & 0.17 \\
\hline Yes & 1.00 (Ref) & $1.27(0.74-2.19)$ & $1.47(0.78-2.78)$ & 0.62 & \\
\hline No & 1.00 (Ref) & $1.18(0.64-2.17)$ & $1.29(0.77-2.16)$ & 0.39 & \\
\hline Histology & & & & & 0.51 \\
\hline Serous & 1.00 (Ref) & $1.19(0.76-1.86)$ & $1.35(0.89-2.05)$ & 0.19 & \\
\hline Non-serous & 1.00 (Ref) & $1.27(0.51-3.17)$ & $1.39(0.48-4.03)$ & 0.77 & \\
\hline Grading & & & & & 0.63 \\
\hline Grade 1 \& 2 & 1.00 (Ref) & $1.07(0.49-2.35)$ & $1.18(0.58-2.40)$ & 0.75 & \\
\hline Grade 3 & 1.00 (Ref) & $1.29(0.89-1.87)$ & $1.60(0.98-2.61)$ & 0.08 & \\
\hline
\end{tabular}

CI, confidence interval; FIGO, International Federation of Gynecology and Obstetrics; HR, hazard ratio; RD, residual disease.

† HRs ( $95 \%$ CIs) for progression-free survival was estimated by using multivariable proportional hazards models which were adjusted for age at diagnosis, FIGO, RD, performance status, ascites, and grading.

\section{Discussion}

To our knowledge, this is one of the limited epidemiological studies reporting the association between TTC and PFS and OS of EOC patients in Asia. In this retrospective cohort study of ovarian cancer survivors, compared with patients with TTC less than 14 days, delayed TTC (more than 28 days) was associated with a significantly worse PFS and OS among patients with advanced stage.

This study adds to the limited evidence of the effect of TTC on survival for ovarian cancer survivors. Of these published studies, some found a benefit for early treatment initiation [12, 14-17, 23], and others failed to show an effect on survival $[11,13,18,19,21$, 
22]. Among these studies with positive findings, two thirds of them are prospective designed studies $(n=4)$. Notably, two prospective cohort studies found a dose-response relationship of aforementioned association [15, 16]. For example, Mahner et al [15] presented the effect of TTC differed significantly for patients with or without RD on the basis of 3326 patients from three prospective randomized phase III trials. A delay of chemotherapy by one week resulted in a $4 \%$ and $9 \%$ decrease of PFS and OS in patients with RD, respectively. However, delayed TTC was significantly associated with later PFS and no effect towards OS in patients with RD. In contrast, the other six studies failed to find an association which might be attributed to limited sample size and different characteristics of ovarian cancer and administered chemotherapy. Additionally, the majority of these studies failed to evaluate whether there was an interaction between clinical predictors and TTC.

Despite previous epidemiological studies generated inconsistent results, earlier TTC may improve survival through several potential biological mechanisms. Data from animal tumor models suggested that the numbers of circulating tumor cells increased as well as the growth of metastatic deposits strengthened after removal of the primary tumor [5, 22, 23, 29-32]. For example, Gunduz et al [5] detected that removal of the tumor could increase tumor growth by shuttling of non-cycling cells in $G_{0}$ phase into the cell cycle. Furthermore, Fisher et al [6] found that the shorter TTC, the more complete is the abrogation of the kinetic changes in distant tumor foci, the more effective becomes the suppression of residual tumor burden, and the more prolonged is the survival through assessing the variation of residual tumor cell kinetics and animal survival in a murine tumor model. Additionally, tumor cells may develop mutations that confer chemo-resistance during the
TTC interval which reduces the response rate of first line chemotherapy.

Our study has several strengthens. This is the one of limited evidence from Asia investigating the association between TTC and PFS and OS of EOC patients. Additionally, the aforementioned association was evaluated in a group of patients who receive homogenous treatments because randomized controlled trial suggest that paclitaxel plus cisplatin versus paclitaxel plus carboplatin are equally effective $[33,34]$. Furthermore, we carried out numerous subgroup analyses stratified by these well-established prognostic factors as well as sensitivity analyses to assess whether the main findings were robust. We used restricted cubic spline function to test the nonlinearity despite no evidence was observed recently.

Limitations of our study include the following. Firstly, the present study is a retrospective observational study dependent on accurate medical records. Potential recall and confounding bias might exist. However, in 2014, the Shengjing Hospital of China Medical University became the only "Dual Seven Level" hospital in China that reached level 7 of electronic medical record System Application Capacity Grading by the Hospital Management Institute of National Health and Family Planning Commission and level 7 (the highest) of electronic medical record Application Evaluation by the American organization Healthcare Information and Management Systems Society, indicating that the information construction of our hospital has been leading this country and has reached the advanced international standard which may minimize the recall bias. Furthermore, we have addressed the confounding bias by adjusting for all relevant covariates and through regression models. Secondly, although intraperitoneal chemotherapy has already been introduced into China, we only included patients received intravenous chemotherapy in the present study because the later administration is more tolerable and convenient in this country [11]. Thirdly, the TTC can be delayed because of different situations in the clinical practice. For example, when patients are referred from other institutions some weeks after surgery; when patients are submitted to aggressive cytoreductive surgery with consequent recovery delay; when patients present low hemoglobin levels and need more time to recover after surgery; and when patients need to be discharged home earlier after surgery to get adjusted to their cancer diagnosis before starting chemotherapy that

Figure 2. Multivariable-adjusted overall survival curves of patients with ovarian cancer by TTC, estimated from a proportional hazards model (adjusted for age at diagnosis, International Federation of Gynecology and Obstetrics, residual disease, performance status, ascites, and grading) by using a direct adjustment method. The red line indicates the TTC less than 14 days, the green line indicates the TTC between 14 to 28 days, and the blue line indicates the TTC more than 28 days. TTC, time to chemotherapy. 
can be administered in an outpatient facility [20]. Since we could not addressed all these concerns in the present study, the findings should be interpreted with cautious. Further prospective and specifically planned studies are warranted to clarify the aforementioned association. Fourthly, relatively shorter follow-up period (median $=2.97$ years) and more patients with missing information in any variables or covariates $(n=460)$ should be a concern in the present study. However, we found no difference between these included patients and excluding ones. Lastly, failure to finish the first round of chemotherapy may not only decrease the survival of ovarian cancer patients but limit the interpretation of the finding. However, this issue may more appear in younger or earlier stage cancer patients. Additionally, compared to other studies, less patients $(n=69)$ were failure to finish the first round.

In conclusion, our study provided evidence that delayed TTC was associated with a significantly poor PFS and OS in EOC with advanced stage. Further prospective studies are warranted to confirm our findings as well as to guide the development of individualized treatment strategies for women with ovarian cancer.

\section{Acknowledgements}

This study was supported by grants from the Natural Science Foundation of China (No. 81602918 to Qi-Jun $\mathrm{Wu}$ and No. 81272873 to Yi-Sheng Jiao), the Doctoral Start-up Foundation of Liaoning Province (No. 201501007 to Qi-Jun Wu), the Younger research fund of Shengjing Hospital (Grant 2014sj09 to Qi-Jun $\mathrm{Wu}$ ), and the Campus Research Fund of China Medical University (No. YQ20170002 to Qi-Jun Wu).

\section{Competing Interests}

The authors have declared that no competing interest exists.

\section{References}

[1] Siegel R, Naishadham D, Jemal A. Cancer statistics, 2013. CA Cancer J Clin 2013; 63: 11-30.

[2] Siegel RL, Miller KD, Jemal A. Cancer statistics, 2016. CA Cancer J Clin 2016; 66: 7-30.

[3] Winter WR, Maxwell GL, Tian C, et al. Prognostic factors for stage III epithelial ovarian cancer: a Gynecologic Oncology Group Study. J Clin Oncol 2007; 25: 3621-7.

[4] Fidler IJ, Ellis LM. The implications of angiogenesis for the biology and therapy of cancer metastasis. Cell 1994; 79: 185-8.

[5] Gunduz N, Fisher B, Saffer EA. Effect of surgical removal on the growth and kinetics of residual tumor. Cancer Res 1979; 39: 3861-5.

[6] Fisher B, Gunduz N, Saffer EA. Influence of the interval between primary tumor removal and chemotherapy on kinetics and growth of metastases. Cancer Res 1983; 43: 1488-92.

[7] Simpson-Herren L, Sanford AH, Holmquist JP. Effects of surgery on the cell kinetics of residual tumor. Cancer Treat Rep 1976; 60: 1749-60.

[8] Kiguchi K, Kubota T, Aoki D, et al. A patient-like orthotopic implantation nude mouse model of highly metastatic human ovarian cancer. Clin Exp Metastasis 1998; 16: 751-6.
[9] Schiffenbauer YS, Abramovitch R, Meir G, et al. Loss of ovarian function promotes angiogenesis in human ovarian carcinoma. Proc Natl Acad Sci U S A 1997; 94: 13203-8.

[10] Bell RS, Roth YF, Gebhardt MC, et al. Timing of chemotherapy and surgery in a murine osteosarcoma model. Cancer Res 1988; 48: 5533-8.

[11] Feng Z, Wen H, Bi R, et al. Prognostic impact of the time interval from primary surgery to intravenous chemotherapy in high grade serous ovarian cancer. Gynecol Oncol 2016; 141: 466-70.

[12] Tewari KS, Java JJ, Eskander RN, et al. Early initiation of chemotherapy following complete resection of advanced ovarian cancer associated with improved survival: NRG Oncology/Gynecologic Oncology Group study. Ann Oncol 2016; 27: 114-21

[13] Lydiksen L, Jensen-Fangel S, Blaakaer J. Is it possible to define an optimal time for chemotherapy after surgery for ovarian cancer? Gynecol Oncol 2014; 133: 454-9.

[14] Heo EJ, Lee YY, Kim TJ, et al. The impact of the interval from surgery to initiation of chemotherapy (ISC) on survival in advanced epithelial of ovarian cancer. Int J Gynecol Cancer 2014; 244: 209.

[15] Mahner S, Eulenburg C, Staehle A, et al. Prognostic impact of the time interval between surgery and chemotherapy in advanced ovarian cancer: analysis of prospective randomised phase III trials. Eur J Cancer 2013; 49: 142-9.

[16] Hofstetter G, Concin N, Braicu I, et al. The time interval from surgery to start of chemotherapy significantly impacts prognosis in patients with advanced serous ovarian carcinoma-analysis of patient data in the prospective OVCAD study. Gynecol Oncol 2013; 131: 15-20.

[17] Wright JD, Herzog TJ, Neugut AI, et al. Effect of radical cytoreductive surgery on omission and delay of chemotherapy for advanced-stage ovarian cancer. Obstet Gynecol 2012; 120: 871-81.

[18] Aletti GD, Long HJ, Podratz KC, et al. Is time to chemotherapy a determinant of prognosis in advanced-stage ovarian cancer? Gynecol Oncol 2007; 104: 212-6.

[19] Paulsen T, Kaern J, Kjaerheim K, et al. Influence of interval between primary surgery and chemotherapy on short-term survival of patients with advanced ovarian, tubal or peritoneal cancer. Gynecol Oncol 2006; 102: 447-52.

[20] Gadducci A, Sartori E, Landoni F, et al. Relationship between time interval from primary surgery to the start of taxane- plus platinum-based chemotherapy and clinical outcome of patients with advanced epithelial ovarian cancer: results of a multicenter retrospective Italian study. J Clin Oncol 2005; 23: 751-8.

[21] Sorbe B. Prognostic importance of the time interval from surgery to chemotherapy in treatment of ovarian carcinoma. Int J Gynecol Cancer 2004; 14: 788-93.

[22] Flynn PM, Paul J, Cruickshank DJ. Does the interval from primary surgery to chemotherapy influence progression-free survival in ovarian cancer? Gynecol Oncol 2002; 86: 354-7.

[23] Warwick J, Kehoe S, Earl H, et al. Long-term follow-up of patients with advanced ovarian cancer treated in randomised clinical trials. Br J Cancer 1995; 72: 1513-7.

[24] Liu Y, Zhang T, Wu Q, et al. Relationship between initiation time of adjuvant chemotherapy and survival in ovarian cancer patients: a dose-response meta-analysis of cohort studies. Sci Rep 2017; 7: 9461.

[25] Eisenhauer EA, Therasse P, Bogaerts I, et al. New response evaluation criteria in solid tumours: revised RECIST guideline (version 1.1). Eur J Cancer 2009; 45: 228-47.

[26] Wu OJ, Yang G, Zheng W, et al. Pre-diagnostic cruciferous vegetables intake and lung cancer survival among Chinese women. Sci Rep 2015; 5: 10306.

[27] Desquilbet L, Mariotti F. Dose-response analyses using restricted cubic spline functions in public health research. Stat Med 2010; 29: 1037-57.

[28] Yang G, Shu XO, Li HL, et al. Prediagnosis soy food consumption and lung cancer survival in women. J Clin Oncol 2013; 31: 1548-53.

[29] Colleoni M, Bonetti M, Coates AS, et al. Early start of adjuvant chemotherapy may improve treatment outcome for premenopausal breast cancer patients with tumors not expressing estrogen receptors. The International Breast Cancer Study Group. J Clin Oncol 2000; 18: 584-90.

[30] Pronzato P, Campora E, Amoroso D, et al. Impact of administration-related factors on outcome of adjuvant chemotherapy for primary breast cancer. Am J Clin Oncol 1989; 12: 481-5.

[31] [No authors listed]. Combination adjuvant chemotherapy for node-positive breast cancer. Inadequacy of a single perioperative cycle. N Engl J Med 1988; 319: 677-83

[32] Buzdar AU, Smith TL, Powell KC, et al. Effect of timing of initiation of adjuvant chemotherapy on disease-free survival in breast cancer. Breast Cancer Res Treat 1982; 2: 163-9.

[33] du Bois A, Luck HJ, Meier W, et al. A randomized clinical trial of cisplatin/paclitaxel versus carboplatin/paclitaxel as first-line treatment of ovarian cancer. J Natl Cancer Inst 2003. 95: 1320-9.

[34] Neijt JP, Engelholm SA, Tuxen MK, et al. Exploratory phase III study of paclitaxel and cisplatin versus paclitaxel and carboplatin in advanced ovarian cancer. J Clin Oncol 2000; 18: 3084-92. 